\title{
Regional Radiation Pneumonitis After SIRT of a Subcapsular Liver Metastasis: What is the Effect of Direct Beta Irradiation?
}

\author{
Tomas Dobrocky • Markus Fuerstner • \\ Bernd Klaeser • Ruben Lopez-Benitez • \\ Yara Banz Wälti · Levent Kara
}

Received: 23 July 2014/ Accepted: 8 September 2014/Published online: 7 November 2014

(C) Springer Science+Business Media New York and the Cardiovascular and Interventional Radiological Society of Europe (CIRSE) 2014

\begin{abstract}
We herein present a patient undergoing selective internal radiation therapy with an almost normal lung shunt fraction of $11.5 \%$, developing histologically proven radiation pneumonitis. Due to a predominance of pulmonary consolidations in the right lower lung and its proximity to a large liver metastases located in the dome of the right liver lobe a Monte Carlo simulation was performed to estimate the effect of direct irradiation of the lung parenchyma. According to our calculations direct irradiation seems negligible and RP is almost exclusively due to ectopic draining of radioactive spheres.
\end{abstract}

Keywords SIRT $\cdot$ Radioembolization · Yttrium-90 · Interventional oncology $\cdot$ Radiation pneumonitis

T. Dobrocky $(\bowtie) \cdot R$. Lopez-Benitez $\cdot$ L. Kara Department of Interventional, Pediatric and Diagnostic Radiology, Inselspital, University Hospital, University of Bern, Freiburgstrasse 10, 3010 Bern, Switzerland

e-mail: tomas.dobrocky@insel.ch

R. Lopez-Benitez

e-mail: ruben.lopez@insel.ch

L. Kara

e-mail: levent.kara@insel.ch

M. Fuerstner · B. Klaeser

Department of Nuclear Medicine, Inselspital, University

Hospital, University of Bern, Freiburgstrasse 10, 3010 Bern,

Switzerland

e-mail: markus.fuerstner@insel.ch

B. Klaeser

e-mail: bernd.klaeser@insel.ch

\section{Y. B. Wälti}

Institute of Pathology, University Hospital, University of Bern, Freiburgstrasse 10, 3010 Bern, Switzerland

e-mail: yara.banz@pathology.unibe.ch

\section{Introduction}

Selective internal radiation therapy (SIRT) is a catheterguided application of small, non-biodegradable radioactive microspheres into tumor supplying branches of the hepatic artery for treatment of primary and metastatic liver cancer, combining embolization of neoplastic microvasculature with intratumoral radiotherapy. Hepatic malignancies derive $80-100 \%$ of their blood supply from the arterial circulation [1], and on single-photon emission computed tomography (SPECT) show a threefold greater vascular density than normal liver parenchyma giving a favorable tumor-to-normal $(\mathrm{T} / \mathrm{N})$ tissue uptake ratio.

Hepatic neoplasms are well known for their neovascularization and may induce arterio-venous shunts, thus allowing quick passage of administered embolic material to the systemic circulation [2]. Mapping of the hepatic vasculature, dose calculation, appraisal of lung shunt fraction (LSF), and embolization of possible hepato-systemic shunts are the main goal of the pretreatment work-up, thus optimizing distribution of microspheres, minimizing radiation damage to uninvolved liver parenchyma and extrahepatic structures after Yttrium-90 microspheres $\left({ }^{90} \mathrm{Y}\right)$ application.

Taking into account patient selection criteria and safety recommendations SIRT is a safe and minimally invasive procedure well tolerated by the patients. However, shortterm complications appearing within a few days after the procedure, including abdominal pain, nausea, emesis, and fever have been described. Long-term complications occurring due to non-target embolization of infused radioactive ${ }^{90} \mathrm{Y}$ microspheres include gastroduodenal ulcers, pneumonitis, hepatitis presenting with ascites, rapid weight gain, liver enlargement, jaundice, and increased transaminase levels, pancreatitis, or cholecystitis. 
A

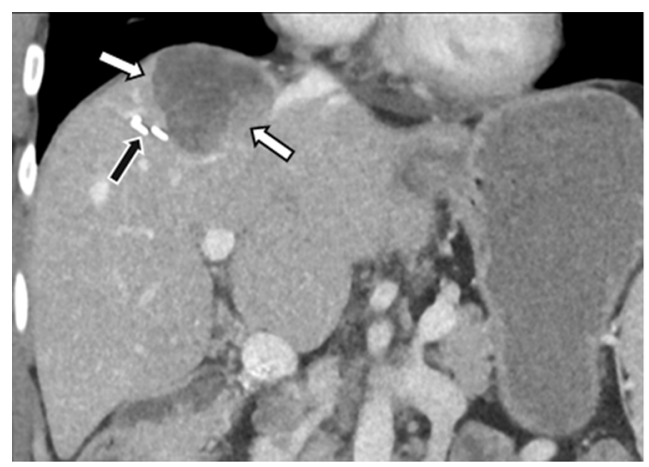

C

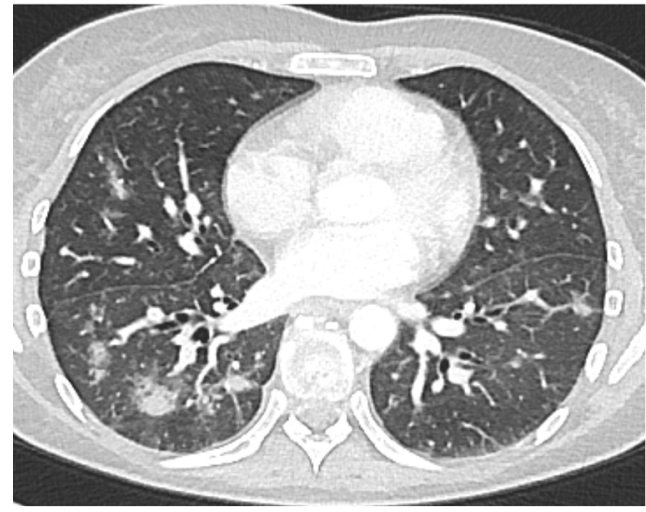

$\mathbf{E}$

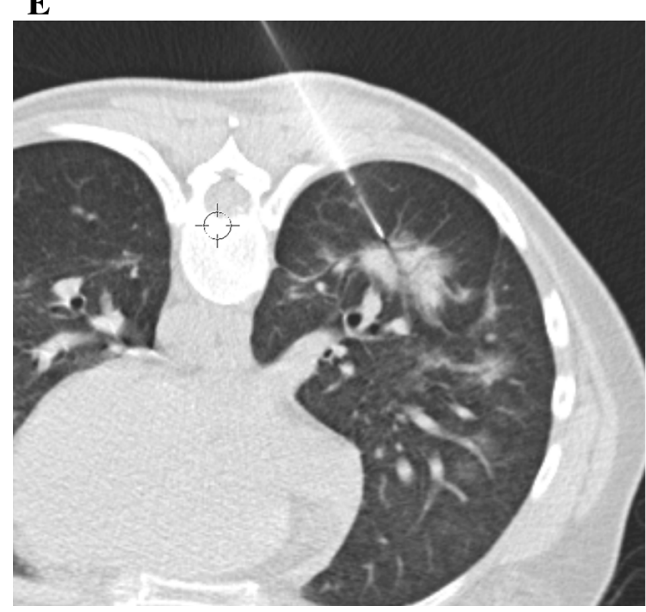

Fig. 1 A and B Coronal and axial CT images 2 months after metastasectomy (metal clips-black arrows) showing recurrent multifocal hepatic metastases (white arrows). No evidence of extrahepatic tumor recurrence. $\mathbf{C}$ and $\mathbf{D}$ Axial and coronal CT images 3 months after SIRT showing multiple, bilateral patchy

\section{Case}

We report the case of a 39-year-old female patient with intra-abdominal leiomyosarcoma extending from the lower border of the right hepatic lobe to the iliac vessels with invasion of the inferior vena cava (IVC). No metastasis was present at the time of initial diagnosis. The patient
B

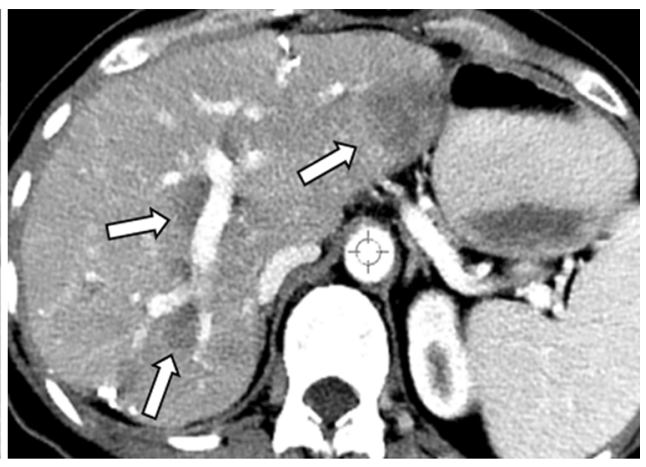

D

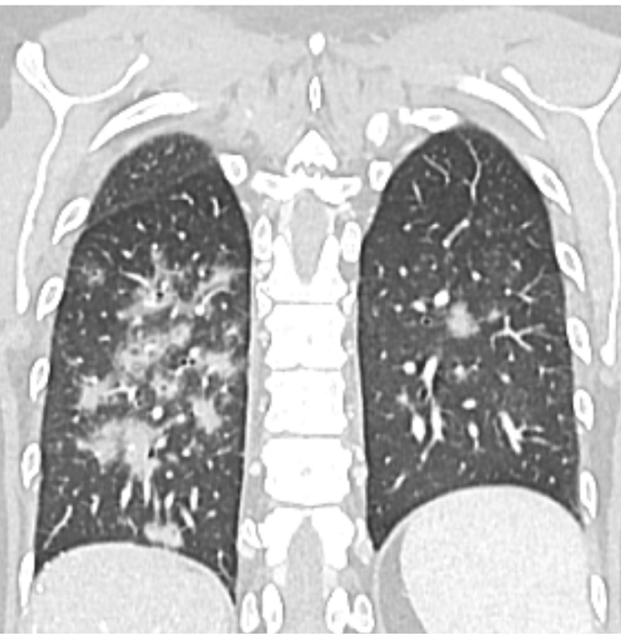

pulmonary consolidations with surrounding ground glass attenuation with an apico-basal gradient predominantly localized in the right lower lobe. E CT-guided lung biopsy of a solid, spiculated lesion in the right lower lobe. Patient in the supine position showing the tip of a 20-Gauge needle within the suspicious lesion

underwent radical tumor resection and reconstruction of the IVC with a dacron bypass.

Computed tomography (CT) one year after initial diagnosis showed multifocal hepatic lesions, metastasis in the lower pole of the right kidney and right ovary. Subsequent atypical hepatectomy, partial nephrectomy, and ovariohysterectomy were performed. Two months after metastasectomy 
A

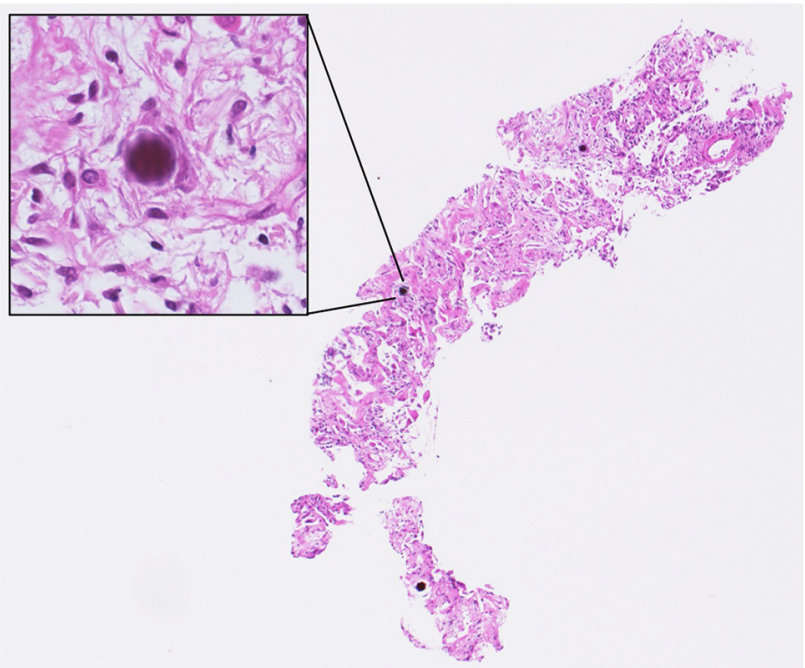

\section{$500 \mu \mathrm{m}$}

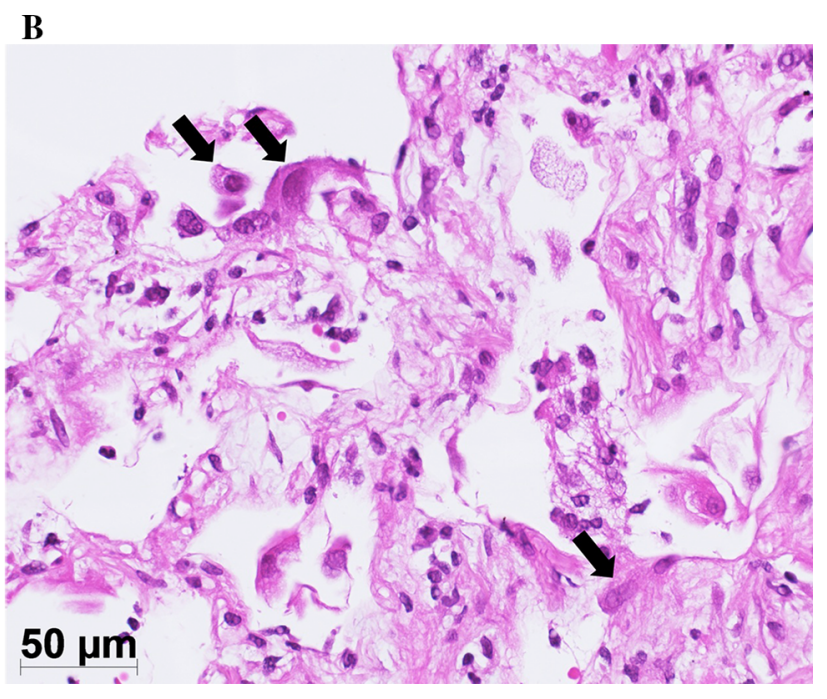

Fig. 2 A Hematoxylin and Eosin staining, overview $(5 \times)$. Lung biopsy in the overview reveals slightly consolidated alveolar parenchyma. In the midst of the biopsy three darkly basophilic/black round particles (roughly 50-60 $\mu \mathrm{m}$ in size) are observed. In the top left inlay is an enlarged image of one of the particles, localized within the interstitial tissue, no longer clearly associated with the vasculature. These particles correspond to the Yttrium-90 microspheres used for SIRT. B Hematoxylin and Eosin staining, detail $(40 \times)$. The black arrows point to atypical alveolar epithelium with enlarged, hyperchromatic nuclei with somewhat "smudgy" chromatin-characteristics compatible with radiogenic alterations. Also, alveolar septae are slightly enlarged with some fibrosis and minimal chronic lymphocytic inflammatory infiltrate

new liver metastases in liver segments II/III, V, VIII (Fig. 1A, B) were detected, without evidence of extrahepatic tumor recurrence. Based on a multidisciplinary tumor board decision, and given the fact that the liver was the major site of disease on restaging, the patient was referred for a SIRT.
Pretreatment transfemoral angiogram of the celiac trunk and superior mesenteric artery showed normal anatomy with no aberrant hepatic supply. After embolization of the gastroduodenal artery in one HydroCoil technique [3], no shunting or collateral flow to the gastrointestinal tract was evident. Tumor supplying branches of the hepatic artery were hypertrophied, demonstrating a tortuous course. Super-selective catheter placement within the right and left hepatic artery with injection of Technetium-99m-labeled macro-aggregated albumin $\left({ }^{99 \mathrm{~m}} \mathrm{Tc}\right.$ MAA) simulating distribution of ${ }^{90} \mathrm{Y}$ microspheres was performed. Subsequent planar scintigraphy demonstrated a lung shunt fraction (LSF) of $11.5 \%$. The dose was calculated according to the body surface area (BSA) method and reduced by $20 \%$ due to increased LSF [4].

Eleven days after the initial work-up, bilobar administration of ${ }^{90} \mathrm{Y}$ resin microspheres (SIRTex Medical, Inc., Lane Cove, New South Wales, Australia) was initiated with matching position of the catheter tip within the right and left hepatic artery as on previous ${ }^{99 \mathrm{~m}}$ Tc MAA infusion. Intermittent injection of microsphere and contrast was performed to verify antegrade flow during application. Total activity administered was $1.60 \mathrm{GBq}$ (right liver lobe 1.1 and left lobe $0.5 \mathrm{GBq}$ ).

The patient was observed for $4 \mathrm{~h}$ in the nuclear medicine suite. As no immediate complications or discomfort was observed, she was discharged on the same day with proton pump inhibitors, steroids, antiemetics, and analgetics in addition to her daily medication.

Three months post-interventionally multiple patchy solid lung opacities with surrounding ground-glass halo were visualized on CT (Fig. 1C, D). The patient was clinically asymptomatic and in particular revealed no symptoms of respiratory discomfort. To rule out pulmonary metastasis a lung biopsy was performed. Histological work-up revealed minimally consolidated lung parenchyma and ${ }^{90} \mathrm{Y}$ microspheres localized mainly within the interstitium (Fig. 2). Six months later the CT showed complete regression of pulmonary consolidations with no additional medical treatment.

\section{Discussion}

SIRT is a minimally invasive treatment option for patients with unresectable primary or secondary liver tumors. The procedure is increasingly implemented in multidisciplinary treatment plans with a growing number of treated patients. As stated by the manufacturer over 25,000 vials of SIRSpheres microspheres have been supplied worldwide so far and thus knowledge of normal findings and possible complication in the post-interventional stage is essential for the radiologist. 
Table 1 Electron ranges for the average and maximum energy of ${ }^{90} \mathrm{Y}$ in different types of tissues

\begin{tabular}{llll}
\hline Energy $(\mathrm{keV})$ & \multicolumn{2}{l}{ CSDA range } & \\
\cline { 2 - 4 } & Air, dry (Near sea level) & Lung tissue (ICRP) & \multicolumn{2}{l}{ Soft tissue (ICRP) } \\
& $\rho=1.2048 \times 10^{-3} \mathrm{~g} \cdot \mathrm{cm}^{-3}(\mathrm{~cm})$ & $\rho=0.3 \mathrm{~g} \cdot \mathrm{cm}^{-3}(\mathrm{~cm})$ & $\rho=1.0 \mathrm{~g} \cdot \mathrm{cm}^{-3}(\mathrm{~cm})$ \\
\hline 926.7 & 371 & 1.3 & 0.3 \\
2279.8 & 1026 & 3.8 & 1.1 \\
\hline
\end{tabular}

Radiation pneumonitis (RP) is a radiation-induced lung inflammation generally occurring two to four months after SIRT. RP after SIRT is a rare adverse reaction with incidence rates ranging from 0 to $6.3 \%[5,6]$. A comprehensive online search using PubMed identified a series by Salem et al. [5], Leung et al. [6], and cases reports by Lin [7] and Wright [8]. In their study, Leung et al. reported that five out of 80 patients undergoing SIRT developed RP, four of these with histologically proven ${ }^{90} \mathrm{Y}$ microspheres on lung biopsy. The estimated whole lung radiation dose ranged from 10.43 to $36.44 \mathrm{~Gy}$. Five out of nine patients with a LSF $>13 \%$ developed RP, whereas RP was not observed with a LSF $<13 \%$ [6]. Salem et al. reported on 403 patients treated with ${ }^{90} \mathrm{Y}$ microspheres, 58 of which received a cumulative lung dose (CLD) of $>30$ Gy. However, not a single patient showed clinical or radiological signs of RP [5].

There are three principles explaining the mechanism of inadvertent irradiation of surrounding tissues in SIRT: (1) Ectope draining of radioactive spheres via shunts (e.g., hepato-pulmonary shunt) to non-target organs, (2) direct irradiation of tissue due to close proximity to the liver, and (3) leaching of radionuclide from the microspheres [9]. Leaching, however, is currently not considered a significant source of radiation dose in glass and resin spheres used clinically [9].

\section{Ectope Draining}

As stated previously, hepatic malignancies are known for their neovascularization and may lead to arterio-venous shunts thus allowing ${ }^{90} \mathrm{Y}$ microspheres applied into the hepatic artery to pass to the lung. The lung possesses a similar capillary structure to the liver and will therefore capture the shunted microspheres. Based on the lung shunt fraction (LSF) determined on ${ }^{99} \mathrm{~m}$ Tc MAA scan the cumulative absorbed radiation dose to the lungs CLD during SIRT can be calculated as follows:

Formula $1: D(\mathrm{~Gy})=50 \times$ lung mass $\sum_{i=1}^{n} A \times \mathrm{LSF}$,

where $A_{i}$ is the activity infused, $\mathrm{LSF}_{i}$ is lung shunt fraction during infusion, $n$ is number of infusions, and lung mass assumed $1 \mathrm{~kg}$. In our case: CLD $(\mathrm{Gy})=50 \times 1 \times 1.6 \times$ $0.115=9.2 \mathrm{~Gy}$.

Due to a fairly low LSF and CLD in our patient (11.5\% and $9.2 \mathrm{~Gy}$, respectively), we estimated the degree of the additional effect of direct irradiation of the lung due to ${ }^{90} \mathrm{Y}$ microspheres lodged within hepatic metastases.

\section{Direct Irradiation}

The asymmetric location of lung consolidation is a finding, potentially supporting the theory of additional irradiation of lung parenchyma due to its proximity to liver metastases embolized with ${ }^{90} \mathrm{Y}$ microspheres. As shown on the coronal CT scan (Fig. 1C, D) there is an apico-basal gradient and a clear predominance of lung consolidations in the right lower lobe bordering the right liver lobe. In fact, the lung base is separated from the largest liver metastasis in the dome of segment VIII (diameter $6 \mathrm{~cm}$ ) just by a thin muscular layer of the diaphragm.

To further investigate the possible effect of direct irradiation, basic principles of physics have to be taken into account. ${ }^{90} \mathrm{Y}$ is a pure beta emitter with an average and maximal energy of 926.7 and $2279.8 \mathrm{keV}$. The continuous slowing down approximation (CSDA) ranges of the electrons in air, lung tissue and soft tissue taken from [10] are given in Table 1.

Estimation of dose distribution in the lung caused by a spherical tumor in the liver was performed with the FLUKA code, a fully integrated particle physics Monte Carlo simulation package, which very accurately simulates electromagnetic and nuclear interactions in matter. Monte Carlo (MC)-Code used: FLUKA 2011.2b.5 [11, 12]. For the MC simulation the International Commission on Radiological Protection (ICRP) composition and density of the lung and soft tissue was used [10]. As radiation source the radionuclide ${ }^{90} \mathrm{Y}$ as implemented in FLUKA was uniformly distributed over the spherical tumor. The transport threshold for electrons was set to $1 \mathrm{keV}$. Eight MC-runs have been conducted, each with $10^{7}$ disintegrations of ${ }^{90} \mathrm{Y}$. The dose distribution may be seen in the color plot below (Fig. 3C).

The pulmonary consolidations seen were located well within the apical parts of the right lower lung lobe, where 
Fig. 3 A Sagittal CT image showing a large liver metastasis with a maximum diameter of $6 \mathrm{~cm}$ located in the dome of the right lobe abutting the diaphragm. B The picture shows the geometry of the MC-

Simulation. The spherical tumor is located in the liver (red), in a distance of $0.1 \mathrm{~cm}$ from the lung (white). C The dose distribution was calculated in a three dimensional mesh of $20 \times 20 \times 20 \mathrm{~cm}^{3}$ with a voxel dimension of

$0.1 \times 0.1 \times 0.1 \mathrm{~cm}^{3}$. Black vertical line symbolizes the diaphragm. Liver parenchyma with a spherical tumor (red) is to the left of the diaphragm, and lung parenchyma to the right. In the tumor a dose of $1.18 \times 10^{-12} \pm 2.85 \times 10^{-14}$ is deposited per disintegration. Assuming a target dose of $200 \mathrm{~Gy}$ in the tumor volume results in $1.7 \times 10^{14}$ disintegrations. The color plot shows the averaged dose distribution over eight slices in the $\mathrm{x}$-direction for $1.7 \times 10^{14}$ disintegrations
A

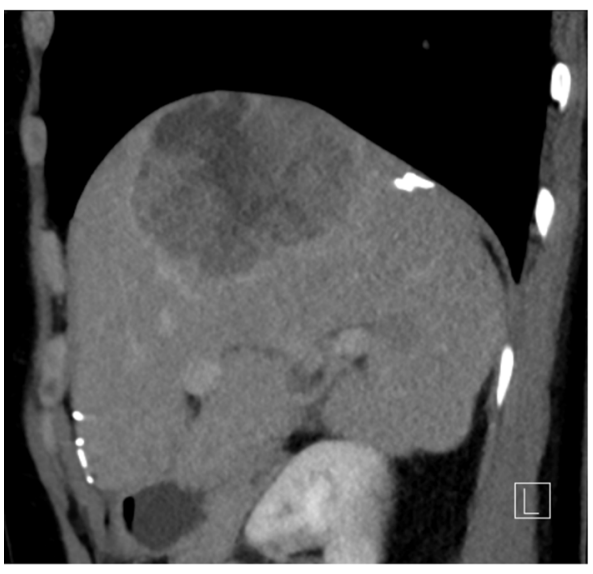

C

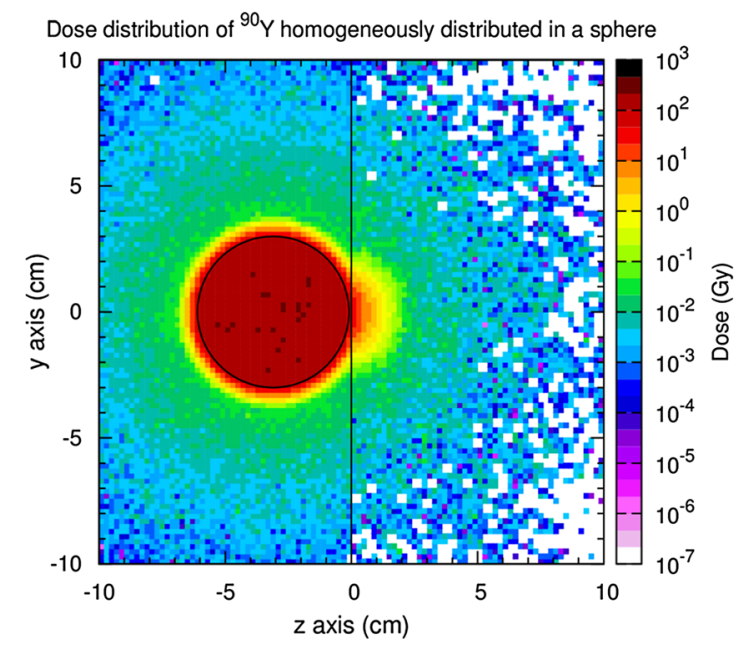

产 (n)
B

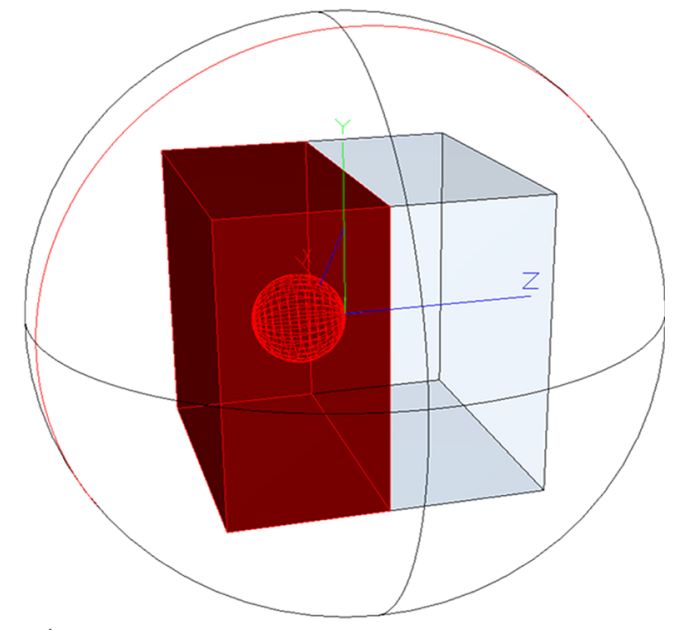

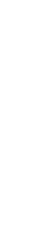

due to the steep drop in dose the additional radiation effect becomes negligible. The most likely explanation why RP occurred in a rather low LSF and despite a dose reduction of $20 \%$, is a high sensitivity of lung parenchyma in our patient. A marked change of LSF between the ${ }^{99 \mathrm{~m}}$ Tc MAA scan and application of radioactive ${ }^{90} \mathrm{Y}$ microspheres 11 days later does not seem a plausible explanation.

\section{Conclusion}

Even in patients with a low CLD, radiation-induced lung injury may develop and is almost exclusively due to ectopic draining of radioactive spheres through hepatopulmonary shunts. According to our calculations, additional effect of direct irradiation of the lung due to ${ }^{90} \mathrm{Y}$ microspheres lodged within treated metastases in the liver dome is negligible.

Due to the increasing number of patients undergoing SIRT, the knowledge of normal findings and possible complications in the post-interventional phase is essential in order to prevent unnecessary invasive work-up (i.e., biopsy).

Acknowledgment This research received no specific Grant from any funding agency in the public, commercial, or not-for-profit sectors.

Conflict of interest Tomas Dobrocky, Markus Fürstner, Bernd Klaeser, Ruben Lopez-Benitez, Yara Banz Wälti, and Levent Kara declare that they have no conflicts of interest.

Statement of informed consent Informed consent was obtained from all individual participants included in the study.

Ethical approval For this type of study formal consent is not required.

\section{References}

1. Kennedy A, Nag S, Salem R et al (2007) Recommendations for radioembolization of hepatic malignancies using yttrium-90 microsphere brachytherapy: a consensus panel report from the radioembolization brachytherapy oncology consortium. Int J Radiat Oncol Biol Phys 68:13-23. doi:10.1016/j.ijrobp.2006.11.060 
2. Leung WT, Lau WY, Ho SK et al (1994) Measuring lung shunting in hepatocellular carcinoma with intrahepatic-arterial technetium-99m macroaggregated albumin. J Nucl Med 35:70-73

3. López-Benítez R, Hallscheidt P, Kratochwil C et al (2013) Protective embolization of the gastroduodenal artery with a oneHydroCoil technique in radioembolization procedures. Cardiovasc Interv Radiol 36:105-110. doi:10.1007/s00270-012-0361-9

4. Lambert B, Mertens J, Sturm EJ et al (2010) 99mTc-labelled macroaggregated albumin (MAA) scintigraphy for planning treatment with $90 \mathrm{Y}$ microspheres. Eur J Nucl Med Mol Imaging 37:2328-2333. doi:10.1007/s00259-010-1566-2

5. Salem R, Parikh P, Atassi B et al (2008) Incidence of radiation pneumonitis after hepatic intra-arterial radiotherapy with yttrium90 microspheres assuming uniform lung distribution. Am J Clin Oncol 31:431-438. doi:10.1097/COC.0b013e318168ef65

6. Leung TW, Lau WY, Ho SK et al (1995) Radiation pneumonitis after selective internal radiation treatment with intraarterial 90yttrium-microspheres for inoperable hepatic tumors. Int $\mathbf{J}$ Radiat Oncol Biol Phys 33:919-924. doi:10.1016/03603016(95)00039-3
7. Lin M (1994) Radiation pneumonitis caused by yttrium-90 microspheres: radiologic findings. AJR Am J Roentgenol 162:1300-1302. doi:10.2214/ajr.162.6.8191985

8. Wright CL, Werner JD, Tran JM et al (2012) Radiation pneumonitis following yttrium-90 radioembolization: case report and literature review. J Vasc Interv Radiol 23:669-674. doi:10.1016/j. jvir.2012.01.059

9. Welsh JS, Kennedy AS, Thomadsen B (2006) Selective Internal Radiation Therapy (SIRT) for liver metastases secondary to colorectal adenocarcinoma. Int J Radiat Oncol Biol Phys 66:S62S73. doi:10.1016/j.ijrobp.2005.09.011

10. Mayles P, Nahum A, Rosenwald JC (2007) Handbook of radiotherapy physics: theory and practice. CRC Press, Boca Raton

11. Battistoni G, Muraro S, Sala PR, et al. (2006) The FLUKA code: Description and benchmarking. In: Albrow M, Raja R (eds) Proc. Hadron. Show. Simul. Work. 2006, Fermilab 6-8 Sept. 2006. pp 31-49

12. Ferrari A, Sala PR, Fasso` A, Ranft J (2005) FLUKA: a multiparticle transport code 